\title{
NEED FOR REVISING PATIENT DOSE PROTOCOLS IN PET MEDICAL IMAGING BASED ON NOVEL TECHNOLOGY IMPROVEMENTS
}

\author{
Vojislav Antic ${ }^{*}$, Julie Haglund²
}

${ }^{1}$ University Clinical Centre of Serbia, Belgrade, Serbia

${ }^{2}$ Medical Physicist, Gothenburg, Sweden

\begin{abstract}
Clinical studies have shown that it is possible to improve image contrast up to $30 \%$ when time of flight (TOF) technology is used compared to non-TOF technology [1]. On the other hand, it has been shown that, combined with the point spread function (PSF) information and current ordered-subset-maximum-likelihood (OSEM) algorithm, image quality for overweight patients could be significantly improved by applying TOF. This encourages further study and a change of the dose model based on patient weight. This change would result in the revision of the existing EANM procedure guidelines, either replacing them or combining them with a system based on a body-mass-index (BMI) concept, and thus result in significant dose reductions with stronger patient-based dose optimisation. This will also reduce exposure of the nuclear medicine staff. Bearing in mind that the compromise in clinical practice is either to reduce the time of the scan or to reduce the patient dose, and that this depends mostly on whether a facility has a medical cyclotron or not, this work focuses on the overview of possibilities for potential reduction of patient dose based on appropriate clinical studies.
\end{abstract}

Key words: Medical imaging, PET, TOF, PSF, BMI

DOI: $10.21175 /$ RadProc.2016.29

\section{INTRODUCTION}

Time of flight (TOF) in Positron Emission Tomography (PET) existed for more than 30 years as a concept, but limitations in hardware and software prevented its routine clinical application [1]. Ten years ago, new scintillator detectors based on lutetium were introduced, combining both high light output and short decay time. These scintillators brought the needed timing resolution for standard whole-body time of flight PET using ${ }^{18} \mathrm{~F}$ [1]. TOF implementation in PET brings coincidence timing resolution to the imaging process. Compared with non--TOF imaging, this additional information reduces the uncertainty and permits images to be created using fewer iterations and yields images with better signal--to--noise--ratio (SNR). Simultaneously were analyzed possibilities of reducing degree of spreading (blurring) of the point object - firstly performed with a point source in a uniform cylinder [2] and afterwards in clinical studies. The symbiosis of these PET image reconstruction approaches, together with the development of detectors and detector blocks, photomultipliers, coincidence processor development, motion management, a variety of specialized application software, etc. indicates the possibilities not only for medical image improvement but also for more sophisticated approaches in determining patient dose, compared to the current recommendations that are based solely on patient weight [3]. In terms of patient dosimetry administration, implementation of TOF technology with PSF mostly implies consideration of the greater impact of the BMI concept, which was even applied in studies on the device/technology with fewer opportunities [4].

\section{INFLUENCE OF TOF AND PSF IN PET MEDICAL IMAGING}

The clinical benefit of TOF and PSF in PET reconstructions is of interest. Improvement from TOF is due to the faster iterative convergence. PSF adds a greater uniform spatial resolution, which enhances small regions of uptake, particularly at large radial distances. These technical improvements must, however, translate into diagnostically useful tools. In oncology, lesion delectability is important in the early diagnosis and staging of patients. Positive patient prognoses are strongly dependent on early detection, so the ability to detect small, low- intensity lesions is critically important [5].

Compared to non--TOF technology, clinical studies have shown that it is possible to improve image contrast up to $30 \%$ when TOF technology is used [6]. In addition to TOF, reconstruction techniques using the point spread function (PSF) improve image quality. 3D PET reconstruction with PSF modeling shows improvement of spatial resolution by $45 \%$ and contrast by $10 \%$ [7]. On the same hand, comparison of PSF with non-PSF images showed a mean bias up to $12 \%$ for SUVmax, 8\% for SUVmean, and 7,5\% for SUVpeak [8]. In addition, it has been shown that, combined with the PSF information and current OSEM algorithms, image quality, especially for overweight patients, could be significantly improved by applying TOF $[5,9]$.

Armstrong et.al. evaluated the effects of using TOF and PSF in reconstruction on values of the SUVmax [10]. Although widely used to define thresholds for malignancy in clinical practice, SUVmax is known to be sensitive to partial volume effects and reconstruction 
parameters. Matching image noise produces marked increases in SUVmax, particularly with PSF reconstructions, and these increases in SUVmax may be clinically significant, depending on local practice. Changes in SUVmax as a consequence of using PSF thus create a challenge to physicians who are accustomed to using SUVmax thresholds to define malignancy.

A trade-off curve of signal enhancement versus noise reduction when using PSF and/or TOF algorithms can be established by applying a range of reconstruction post-filters. It is possible to match SUVmax from PSF--based reconstruction with traditional non--PSF algorithms by applying a specific post-filter, but a better solution might be to find different metrics than SUVmax. Two metrics of interest are SUVmean based on a 50\% isocontour of SUVmax and the total lesion glycolysis. The use of total lesion glycolysis (TLG) has been reported in assessment of therapy response and for prognosis $[3,10]$. The increased stability of TLG with a volume delineation based on a percentage of SUVmax suggests that this metric may be more appropriate than SUVmax for staging and prognosis.

\section{Potential of TOF and PSF in Patient Dose ADMINISTRATION BASED ON BMI}

Without the possibility of TOF reconstruction, Geismar et.al. studied the impact of reduced administered activity protocols on image quality using only OSEM and found a stronger than linearly increased attenuation of photon transmission in overweight patients [11]. It was concluded that administered activity significantly predicts image quality and SNR. In order to maintain diagnostic image quality, a low dose protocol is recommended only for patients with BMI $<22 \mathrm{~kg} / \mathrm{m}^{2}$ [11]. The group acknowledges that technology improvements such as TOF and different reconstruction parameters may improve image quality even when a low dose protocol is applied to overweight patients.

Numerical observers were used to evaluate the relative benefit of TOF + PSF in the clinical data for different patient sizes, lesion contrasts, and scan times. Each reconstruction set was processed with the number of iterations found to maximize SNR. Two schemes were used, data from 3-minutes scans and data using lesions with $60 \mathrm{~K}$ counts. The greatest improvement was clearly seen in the faintest lesions and in the shortest scans [5]. In studying the gain in observer performance for patients with low and high BMI, TOF showed more improvement than PSF. For all iterations of each respective algorithm, the gain of PSF, TOF, and TOF+PSF was higher for the overweight patients [5]. This means that there is a clinical alternative to simply injecting more activity into overweight patients. Applying technology improvements TOF and PSF, the overweight patients can be administered lower activity or an activity equivalent to normal weight patients and still yield images that are clinically useful.

The study by Taniguchi et. al. evaluated image quality in four distinct reconstructions: OSEM alone, OSEM + PSF, OSEM + TOF, and OSEM + PSF + TOF [9]. The combination OSEM + PSF + TOF decreased the coefficient of variance of the liver for overweight patients to that for normal weight patients while increasing the SNR similarly between two patient groups. In both normal and overweight patients, the SNR was significantly increased by applying PSF and TOF. The highest SNR was attained in images reconstructed with OSEM + PSF + TOF. This method of reconstruction was thus found to improve the image quality for overweight patients [9]. Although image quality is improved, clinical usefulness of images depends on lesion detectability, and the research does not indicate that improved image quality due to PSF and TOF translates into clinical usefulness. The SNR in relation to the body weight was studied in both normal and overweight groups. For both groups, the SNR was significantly increased by PSF and by TOF. The highest SNR was observed in images reconstructed with OSEM + PSF + TOF, and the degree of improvement in SNR for the overweight group was not significantly different from that for the normal weight group [9].

Akamatsu et.al. also studied the effects of TOF and PSF on image quality using the same four combinations of reconstruction techniques, OSEM, OSEM + PSF, OSEM + TOF, and OSEM + PSF + TOF [12]. Image quality of clinical images was evaluated using SNR of the liver, and the OSEM + PSF + TOF reconstruction was found to improve SNR of the liver by $24.9 \%$ [12]. The quality of clinical PET images is strongly influenced by patient weight, acquisition time, and injected dose. The reconstruction algorithms with PSF and TOF improve SNR of the liver, indicating that the combination PSF + TOF has the potential to maintain image quality for lower activity objects and shorter acquisition times [12]. In other words, good clinical image quality can be attained for a lower activity or a shorter acquisition time, thus improving patient comfort and reducing the radiation burden for patients and nuclear medicine staff.

A 2015 study by a group in Valencia, Spain investigated the effect of reducing the dose based on BMI instead of patient weight while preserving image quality [6]. With fully $3 \mathrm{D}$ TOF, an administered activity injection table based on BMI was created, with 203.5 $\mathrm{MBq}$ as the lower limit for obtaining the best diagnostic image quality. A reduction of between $9 \%$ and $22 \%$ in administered dose per patient was achieved for the BMI-based dose scheme.

Accounting for technological improvements, it is possible to revise the weight-based activity protocols and reduce administered activities while preserving image quality. This revision makes it possible for facilities without a medical cyclotron to be more effective in their use of FDG as well as to reduce exposure of the nuclear medicine staff. Indeed, an exploratory protocol optimisation is being evaluated by the EARL initiative of EANM [13]. This procedure would allow lowering the administered FDG activity for PET/CT systems with higher sensitivity or improved performance using new, enhanced technology such as better TOF performance, high definition reconstruction techniques, continuous bed motion, or extended axial FOV, which increases system sensitivity. The suggested procedure optimises and determines lower limits for FDG activity for PET/CT systems with respect to recovery factors and SNR [13].

\section{CONCLUSION}

In modern TOF PET scanners, there is an improved trade-off between lesion contrast, image noise, and 
total imaging time, leading to a combination of improved lesion detectability, reduced scan time or injected dose, and more accurate and precise lesion uptake measurement. The benefit is a higher for overweight patients, which leads to a more uniform clinical performance over all patient sizes [14], keeping the administered activity within national limitations and improving the efficiency of clinical schedules.

Technology improvements in PET, primarily the TOF architecture with application of PSF in image reconstruction, encourages further investigations on the modification of the method to select administered activity for the particular patient. This change would result in a revision of the existing EANM procedure guidelines; either replacing them or combining them with a system based on a BMI concept, and thus result in significant dose reductions with stronger individualisation, e.g. patient--based dose optimisation. Owing that the staff is exposed mainly to radiation originating from the patient's body, this approach will also reduce exposure of the nuclear medicine staff.

Acknowledgement. This paper is a collaboration and is entirely the work of the co-authors.

\section{REFERENCES:}

1. S. Surti, "Update on Time-of-Flight PET Imaging," $J$. Nucl. Med., vol. 56, no. 1, pp. 98-105, Jan. 2015

2. J. Qi, R.M. Leahy, S.R. Cherry, A. Chatziioannou and T.H. Farquhar, "High-Resolution 3D Bayesian Image Reconstruction Using the MicroPET Small-Animal Scanner,” Phys. Med. Biol., vol. 43, no. 4, pp. 1001-1013, 1998

3. R. Boellaard et al., "FDG PET and PET/CT: EANM Procedure Guidelines for Tumour Imaging: Version 2.0," Eur. J. Nucl. Med. Mol. Imag., vol. 37, pp. 328-354, Feb. 2015

4. M.H. Mahmud, A.J. Nordin, F.F. Ahmad Saad, A.Z. Azman, "Impacts of Biological and Procedural Factors on Semiquantification Uptake Value of Liver in Fluorine-18 Fluorodeoxyglucose Positron Emission Tomography/Computed Tomography Imaging," Quant. Imag. Med. Surg., vol. 5, no. 5, pp. 700-707, Oct. 2015

5. R. Boellaard, A.T. Willemsen, B. Arends, E.P. Visser, "EARL Procedure for Assessing PET/CT System Specific Patient FDG Activity Preparations for Quantitative FDG PET/CT Studies"

Retrieved from:

http://earl.eanm.org/html/img/pool/EARL-procedure-f or-optimizing-FDG-activity-for-quantiative-FDGPET-st udies version 1 1.pdf

Retrieved on: Feb. 20, 2016
6. R. Sanchez-Jurado, M. Devis, R. Sanz, J. E. Aguilar, M del Puig Cózar and J. Ferrer-Rebolleda, "Whole-Body PET/CT Studies with Lowered 18F-FDG Doses: The Influence of Body Mass Index in Dose Reduction," $J$. Nucl. Med. Technol., vol. 42, no. 1, pp. 62-67, Mar. 2014

7. Y.S. Lee, J.S. Kim, K.M. Kim, J.H. Kang, S.M. Lim, H.-J. Kim, "Performance Measurement of PSF Modeling Reconstruction (True X) on Siemens Biograph TruePoint TrueV PET/CT," Ann. Nucl. Med., vol. 28, no.4, pp. 340348, May 2014

8. S. Sheikhbahaei, C. Marcus, R. Wray, A. Rahmim, M.A. Lodge and R.M. Subramaniam, "Impact of Point Spread Function Reconstruction on Quantitative 18F-FDGPET/CT Imaging Parameters and Inter-Reader Rreproducibility in Solid tumors," Nucl. Med. Commun. vol. 37, no. 3, pp. 288-296, Mar. 2016

9. T. Taniguchi et al. "Improvement in PET/CT Image Quality in Overweight Patients with PSF and TOF," Ann. Nucl. Med., vol. 29, no. 1, pp. 71-77, Jan. 2015

10. I.S. Armstrong, M.D. Kelly, H.A. Williams and J.C. Matthews, "Impact of Point Spread Function Modelling and Time of Flight on FDG Uptake Measurements in Lung Lesions Using Alternative Filtering Strategies," EJNMMI Phys., vol. 1, no. 1, p. 99, Dec. 2014

11. J.H. Geismar et al., "Intra-Individual Comparison of PET/CT with Different Body Weight-Adapted FDG Dosage Regimens," Acta Radiol. Open, vol. 4, no. 2, pp. 1-9, Feb. 2015

12. G. Akamatsu et al., "Improvement in PET/CT Image Quality with a Combination of Point-Spread Function and Time-of-Flight in Relation to Reconstruction Parameters," J. Nucl. Med., vol. 53, no. 11, pp. 1716-1722, Nov. 2012

13. J. Schaefferkoetter, M. Casey, D. Townsend and G. E Fakhri, "Clinical Impact of Time-of-Flight and Point Response Modeling in PET Reconstructions: A Lesion Detection Study," Phys. Med. Biol., vol. 58, no. 5, pp. 1465-1478, Mar. 2013

14. Y. Masuda, C. Kondo, Y. Matsuo, M. Uetani and K. Kusakabe, "Comparison of Imaging Protocols for $18 \mathrm{~F}$ -FDG PET/CT in Overweight Patients: Optimizing Scan Duration Versus Administered Dose," J. Nucl. Med., vol. 5o, no. 6, pp. 844-848, June 2009 